\title{
An Information Theoretic Approach to Adaptive System Training Using Unlabeled Data
}

\author{
Kyu-Hwa Jeong, Jian-Wu Xu, Jose C. Principe \\ Computational NeuroEngineering Laboratory \\ Department of Electrical and Computer Engineering,University of Florida \\ Gainesville, FL 32611 U.S.A. \\ E-mail: \{khjeong, jianwu, principe $\} @$ cnel.ufl.edu
}

\begin{abstract}
Traditionally, supervised learning is performed with pairwise input-output labeled data. After the training procedure, the adaptive system weights are fixed and the system is tested with unlabeled data. Recently, exploiting the unlabeled data to improve classification performance has been proposed in the machine learning community. In this paper, we present an information theoretic approach based on density divergence minimization to obtain an extended training algorithm using unlabeled data during testing. The simulations for classification problems suggest that our method can improve the performance of adaptive system in the application phase.
\end{abstract}

\section{INTRODUCTION}

Supervised learning, including system identification and regression, is performed with input-output labeled data using linear and nonlinear system topologies and optimal criteria based on statistics of the error between the desired samples and system output. The purpose of learning is to extract as much information as possible from the labeled training data to obtain optimal system weights so that they generalize in unlabeled data (typically by splitting the data set into training and testing sets). Once the system is trained, there is no further optimization carried out over the unlabeled data during the actual application (testing) phase [1], [2]. This approach is considered natural to all since we do not have the labels for the testing data to further train the adaptive system.

Due to the fact that labeled samples are much more expensive to collect when compared with unlabeled samples, researchers in machine learning are proposing ideas of learning from unlabeled data. The goal is to design classifiers that can utilize the information present in both the labeled as well as in unlabeled data. One method is transductive inference using support vector machines proposed by Vapnik et al. [3], [4]. Transduction goes from particular (past) samples to particular (future) samples without any attempt to generalize. Another prominent approach is active learning where the learner can 'ask' the expert for a label of a sample in contrast to normal (passive) machine learning where the learner is presented with a static set of examples used to construct a model [5]. There are also some other methods in the literature such as the EM algorithm in a maximum likelihood framework [6], [7]; Smoother function approximation of unlabeled data using the representer theorem in the context of regularization [8]. Recently, Erdogmus et al. introduced an information theoretic framework based on Kullback-Leibler divergence minimization for training adaptive systems in supervised learning settings using both labeled and unlabeled data [9].

In this paper, we propose the Euclidean distance based probability density function (pdf) matching algorithm to extend adaptive system training even after supervised learning is completed. We use the information of the desired signal from the training data and the unlabeled novel testing data to adjust the classifier weights. One big advantage of this new information theoretic approach is the simplicity of computation. We also elucidate the circumstances under which our method can improve the performance of classification by using the unlabeled data in the proposed information theoretic framework.

The paper is organized as follows. First, we give the description of the problem. Then the Euclidean distance based probability density function matching algorithm is presented in section 3. Next, we discuss the conditions under which our method might improve the performance of classification. In order to test our algorithm, we apply our method to an artificial data set and a real biomedical data set in section 5 .

\section{PRoblem Description}

Consider a general function approximation problem. Suppose we have input-output data $\left\{\left(u_{1}, y_{1}\right), \ldots,\left(u_{N}, y_{N}\right)\right\}$ available from an unknown nonlinear function as follow:

$$
d=f(\mathbf{u})+n
$$

The observed output (desired response) $d$ is called the label of the input signal $\mathbf{u}$ and $n$ is corrupted noise. In function approximation, the labels are continuous-valued while in classification problem, the labels are discrete. The goal of supervised learning is to construct an adaptive system with input signal $\mathbf{u}$, output $y$, and weights $\mathbf{w}$ to approximate the function $f$ or classify the output into different categories.

$$
y=g(\mathbf{u}, \mathbf{w})
$$

The adaptive system could be a linear filter $\left(y=\mathbf{w}^{T} \mathbf{u}\right)$, a neural network, or any other topology. The process of supervised learning seeks to optimize the weights to extract relevant information from input-output sample pairs for a specific task. The optimization is carried out by minimization 
or maximization of an optimality criterion. Typically the mean square error (MSE) [1], [2] is used, however there are also alternative selections such as minimum error entropy [10] or the $\epsilon$-insensitive loss function [11]. The error signal is defined as the difference between the available desired output and the output produced by the adaptive system for a particular input $(e=d-y)$.

In the training phase, the adaptive system weights are adjusted to obtain an approximation to $f$ or classify the input data into different categories by minimizing the error. In the application (testing) phase, the weights are fixed and the trained system is tested on novel unlabeled input data $\left\{u_{N+1}, \ldots, u_{K}\right\}$. The probability distributions of the input signal for training $\left\{u_{1}, \ldots, u_{N}\right\}$ and for testing $\left\{u_{N+1}, \ldots, u_{K}\right\}$ can be the same or different. The following question is to be answered in this paper: How can we continue updating the weights of the adaptive system in the application phase and under what conditions can we improve performance?

\section{Euclidean Distance PDF Matching Algorithm}

Our idea is to combine the unlabeled input data in the testing set and the information of the desired output for training in order to continue adjusting the system weights. We assume here that the a priori probability of each class during testing is the same as for training. We will discuss more details about this hypothesis in the next section. Based on this assumption, the Euclidean distance pdf matching algorithm is proposed to adapt the system weights in application phase as

$$
\min _{\mathbf{w}} \int\left(f_{d t r n}(x)-f_{y t s t}(x)\right)^{2} d x
$$

where $f_{d t r n}(x)$ is the pdf of desired signal during training phase, $f_{y t s t}(x)$ is the pdf of system output signal during testing phase and $\mathbf{w}$ is the weight vector of the adaptive system. The Euclidean distance pdf matching cost function minimizes the pdf divergence between the training desired signal and the testing output signal based on the Euclidean distance between the pdfs. In other words, we create a desired signal for the input data during testing by utilizing the pdf information of the desired signal encountered during training. This is also the reason why we must require the same $a$ priori probability among training and testing. Instead of setting the problem in the input data space directly, the Euclidean distance pdf matching criterion utilizes the information at the system output, which avoids the curse of dimensionality. Next we derive a gradient descent algorithm for the cost function in (3).

We propose to compute the Euclidean distance pdf matching cost function directly from data samples, i.e. nonparametrically. This requires a smooth (i.e., continuous and differentiable) estimator for the two probability density functions $f_{d t r n}(x)$ and $f_{y t s t}(x)$. Parzen windowing is a suitable method, which is an asymptotically unbiased and consistent pdf estimator [12]. Given N independent and identically distributed (iid) samples $\left\{x_{1}, \ldots, x_{N}\right\}$, the pdf can be approximated by

$$
\hat{f}_{x}(\xi)=\frac{1}{N} \sum_{i=1}^{N} \kappa\left(\xi-x_{i}, \sigma^{2}\right)
$$

where $\kappa\left(\cdot, \sigma^{2}\right)$ is typically a zero-mean Gaussian kernel with standard deviation $\sigma$. One of the advantages of Parzen window with Gaussian kernel is that we can avoid the integral computation directly, since the integral of two Gaussian kernels generates another Gaussian kernel with different double standard deviation. For convenience, we derive the algorithm in one dimension, but this can be easily extended to multidimensional cases.

$$
\begin{aligned}
J(\mathbf{w})= & \int\left(\hat{f}_{d t r n}(x, d)-\hat{f}_{y t s t}(x, y)\right)^{2} d x \\
= & \int \hat{f}_{d t r n}^{2}(x, d) d x-2 \int \hat{f}_{d t r n}(x, d) \hat{f}_{y t s t}(x, y) d x \\
& +\int \hat{f}_{y t s t}^{2}(x, y) d x \\
= & J_{0}+J_{1}(\mathbf{w})+J_{2}(\mathbf{w})
\end{aligned}
$$

where

$$
\begin{aligned}
J_{0} & =\frac{1}{N^{2}} \sum_{i=1}^{N} \sum_{j=1}^{N} \kappa\left(d_{i}-d_{j}, 2 \sigma^{2}\right) \\
J_{1}(\mathbf{w}) & =-\frac{2}{N M} \sum_{i=1}^{N} \sum_{j=1}^{M} \kappa\left(d_{i}-y_{j}(\mathbf{w}), 2 \sigma^{2}\right) \\
J_{2}(\mathbf{w}) & =\frac{1}{M^{2}} \sum_{i=1}^{M} \sum_{j=1}^{M} \kappa\left(y_{i}(\mathbf{w})-y_{j}(\mathbf{w}), 2 \sigma^{2}\right)
\end{aligned}
$$

$J_{0}$ is not a function of $\mathbf{w}, d$ is the desired training phase signal, $N$ is the number of training samples and $y$ is the system output during testing phase with $M$ samples. Only $J_{1}$ and $J_{2}$ are functions of $\mathbf{w}$ through $y=g(\mathbf{u}, \mathbf{w})$. In order to adjust the weights in the testing phase, we take the derivative of $J$ with respect to $\mathbf{w}$ to obtain the gradient descent update

$$
\mathbf{w}_{\text {new }}=\mathbf{w}_{\text {old }}-\eta \nabla J(\mathbf{w})
$$

where the gradient is evaluated from

$$
\begin{aligned}
\nabla J(\mathbf{w}) & =\nabla J_{1}(\mathbf{w})+\nabla J_{2}(\mathbf{w}) \\
\nabla J_{1}(\mathbf{w}) & =\frac{2}{N M} \sum_{i=1}^{N} \sum_{j=1}^{M} \kappa^{\prime}\left(d_{i}-y_{j}, 2 \sigma^{2}\right) \frac{d y_{j}}{d \mathbf{w}} \\
\nabla J_{2}(\mathbf{w}) & =\frac{1}{M^{2}} \sum_{i=1}^{M} \sum_{j=1}^{M} \kappa^{\prime}\left(y_{i}-y_{j}, 2 \sigma^{2}\right) \frac{d\left(y_{i}-y_{j}\right)}{d \mathbf{w}}(8)
\end{aligned}
$$

A batch method is used here to compute the weights update. An online approach is also possible with the introduction of stochastic information gradient [13]. 


\section{DISCUSSION}

Our methodology requires that the a priori probability of each class remains the same during the training and application phase, but it allows for slight differences between the pdf of training and testing. To examine further, we employ the Bayesian method to find out under what conditions performance can be improved.

Consider a classification problem with two classes, $C_{1}$ and $C_{2}$ with the a priori probability $P\left(C_{1}\right)$ and $P\left(C_{1}\right)$ respectively. The optimal discriminant function separating the two classes is given by

$$
P\left(y=C_{i} \mid \mathbf{u}\right)=\frac{P\left(\mathbf{u} \mid y=C_{i}\right) P\left(y=C_{i}\right)}{P(\mathbf{u})}, i=1,2
$$

Since we use the probability of output data for training as a pseudo desired signal in testing phase in order to continue updating the classifier weights, we have to assume that priors do not change from the training to the testing set, i.e. $P\left(C_{i}\right.$; training $)=P\left(C_{i}\right.$; testing $)$ for $i=1,2$. In the case that the likelihood functions $P\left(\mathbf{u} \mid y=C_{i}\right)$ change from training to the testing set, obviously the optimal decision boundary obtained from training set will not be the optimal for testing set. Under this condition, our algorithm will adjust the decision boundary to a better position such that the correct classification probability increases. In the case that the likelihood functions do not change from the training to the testing set, then according to the Bayesian equation (9) the optimal decision boundary obtained from training phase will remain optimal, since the value of the a posterior probability remains the same. Then there is no need to apply our algorithm. We will illustrate these two cases in the next simulation section.

\section{Simulation Results}

In this section, we will give the simulation results of Euclidean distance pdf matching algorithm applied to adaptive systems in a simple pattern classification problem as well as a real biomedical classification problem. In the simulation, we used the same neural network topology for training and testing. The objective of the experiment is to distinguish (classify) two classes of overlapping two-dimensional patterns labeled class 1 and class 2. Let $C 1$ and $C 2$ denote the set of events for which a random vector $\mathbf{u}$ belong to Class 1 and 2, respectively.

In the first simulation, we artificially generated two classes with conditional pdf for class $1, P\left(\mathbf{u} \mid C_{1}\right)$, being a Gaussian distributed with zero mean and unit variance; the conditional pdf for class 2, $P\left(\mathbf{u} \mid C_{2}\right)$, has mean vector $[2,2]$ and unit variance respectively. The two classes have equal a priori probabilities.

In order to illustrate the effectiveness of our algorithm, we simulated two cases. In the first case, the conditional distributions of the testing pattern 1 and 2 are the same as those of the training patterns. Ideally, the decision boundary will not change after we apply our algorithm since the decision boundary is optimal both for training and testing data sets. But due to the finite sample data effects, the likelihood functions

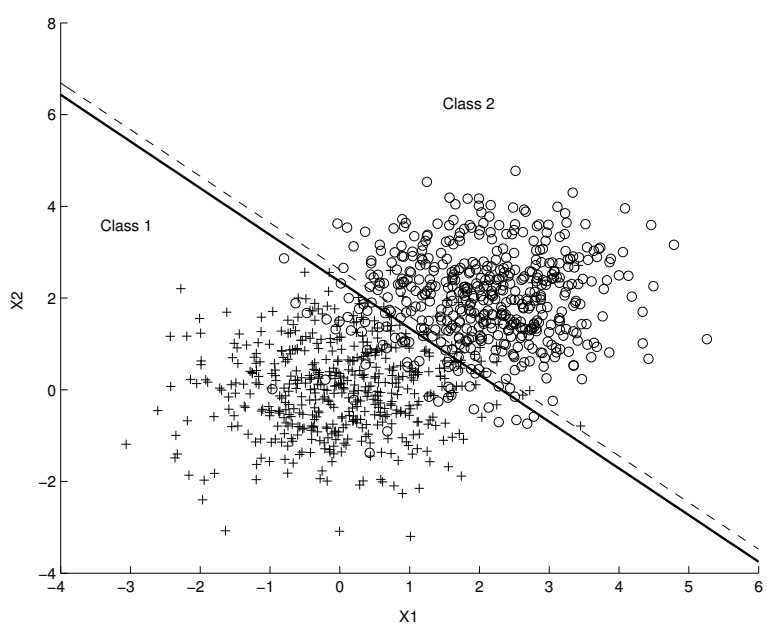

Fig. 1. Two decision boundaries with and without continuous training in testing data set in the case $P\left(\mathbf{u} \mid C_{i} ;\right.$ training $)=P\left(\mathbf{u} \mid C_{i}\right.$; testing $)$ for simulation 1

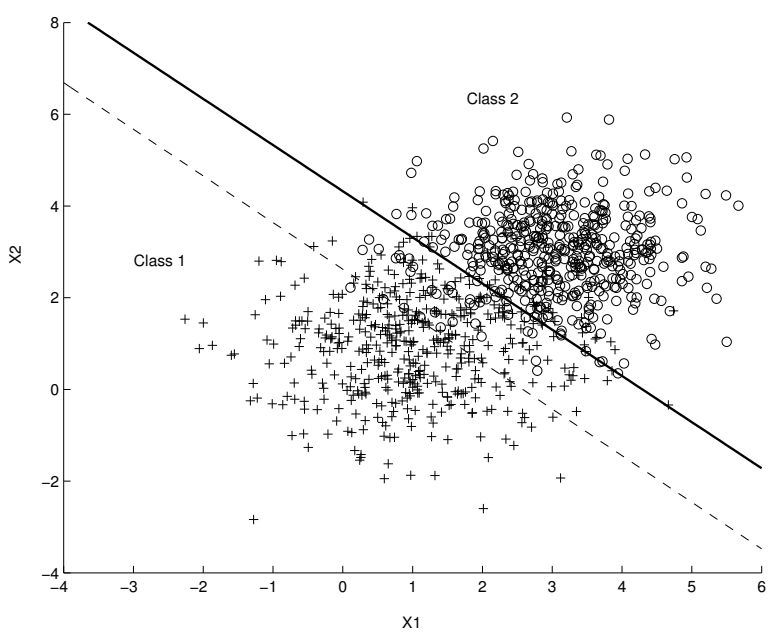

Fig. 2. Two decision boundaries with and without continuous training in testing data set in the case $P\left(\mathbf{u} \mid C_{i} ;\right.$ training $) \neq P\left(\mathbf{u} \mid C_{i} ;\right.$ testing $)$ for simulation 1

will differ slightly for both simulations and the decision boundary will not be optimal during testing. In Fig. 1, the dashed line is the decision boundary for training data set and the solid line is the one after we applied the algorithm for the unlabeled data set (this assignment will be kept for all other figures). The figure shows that the decision boundary resulted from training data moves slightly in the testing data after we applied our algorithm. The correct classification probability increases from 0.905 to 0.919 by using our algorithm. In this situation, our algorithm is able to improve generalization by counteracting overfitting.

In the second case, we generated another testing data set of slightly different likelihood functions compared with training data to mimic the normal variability between experimental conditions. For the testing data class 1 is a gaussian with 


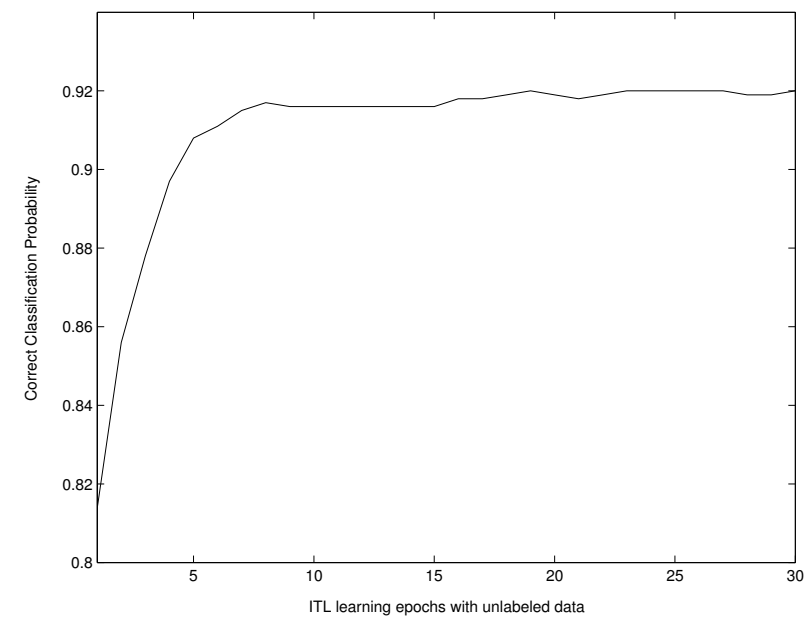

Fig. 3. Correct classification probability curve in the case $P(\mathbf{u}$ $C_{i} ;$ training $) \neq P\left(\mathbf{u} \mid C_{i} ;\right.$ testing $)$ for simulation 1

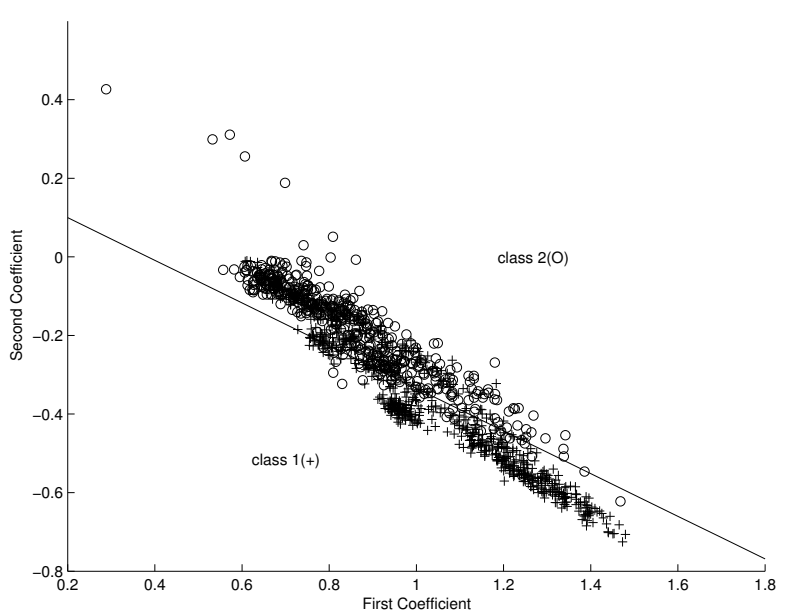

Fig. 4. Training data set and decision boundary in simulation 2

mean vector $[0.2,0.2]$ and variance vector $[1.2,1.2]$, while class 2 is a gaussian with mean vector $[2.1,2.1]$ and variance vector $[0.8,0.8]$. The results are given in fig. 2 and 3. Fig. 2 plots the two decision boundaries with and without continuous training presented in the testing data set. We can see that the decision boundary shifts to a new position where the correct classification probability increases. Fig. 3 gives the correct classification probability curve as a function of iterations. The correct classification probability increases from 0.8 to 0.92 by applying our algorithm.

In the second simulation, we apply the Euclidean distance pdf matching algorithm to the classification of a biomedical data set. This data set was obtained from neural recordings in the surgical treatment of Parkinson's disease. Spike trains are collected from thalamic (Thal) and subthalamic nucleus (STN) cellular activity using deep brain stimulation. The objective of classification is to distinguish the two classes Thal and STN. As a preprocessor, a second order autoregressive (AR) model is applied to segments of the neural activity, where the details

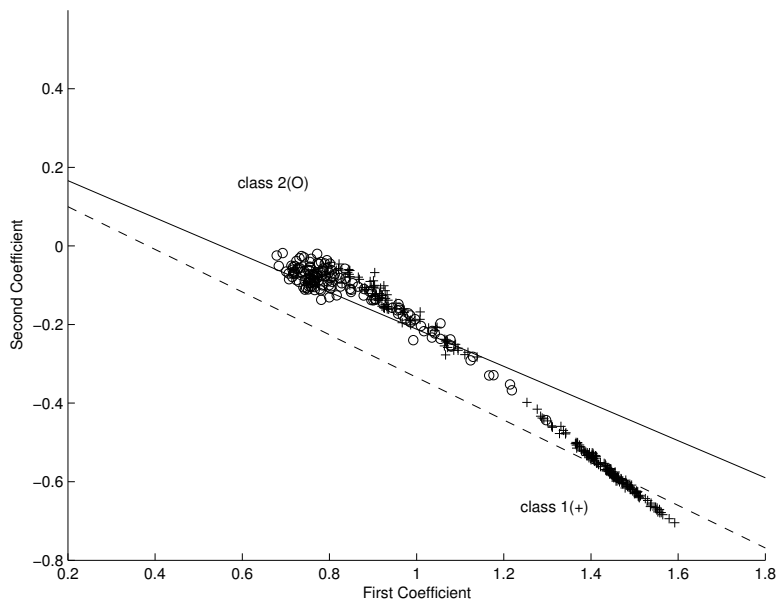

Fig. 5. Two decision boundary with and without continuous training in testing data set for patient 1 in simulation 2

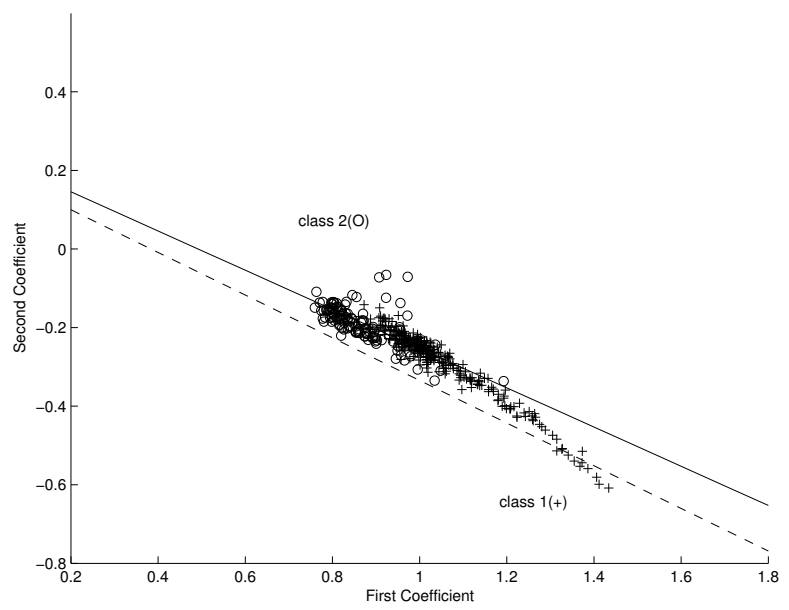

Fig. 6. Two decision boundary with and without continuous training in testing data set for patient 2 in simulation 2

are fully given in [14]. We created the testing data to comply with the hypothesis of equal a priori probabilities of each class as required by our method.

The classifier uses the weights of the AR model. Since Thal and STN signals are different across patients, the conditional probability functions for training and testing will differ. The simulation results are presented in fig. 4 to 7 with class 1 for STN and class 2 for Thal. Fig. 4 shows the scatter plot of the two classes and the decision boundary obtained from a perceptron in the training set comprising the three patients. The total number of training set is 12198 samples and the correct classification probability after the conventional training is 0.7537 . Then we tested our algorithm in each of the different patients and the results are given in fig. 5,6 and 7 respectively. The number of testing set in each test patients are 1976, 2148 and 4086 samples respectively. Since the distributions of each test patients are different from that of the training set, the classification performance in the testing phase is not good as much as training result. It is worth mentioning 




Fig. 7. Two decision boundary with and without continuous training in testing data set for patient 3 in simulation 2

TABLE I

CORRECT CLASSIFICATION PROBABILITY WITH AND WITHOUT CONTINUOUS TRAINING

\begin{tabular}{|c|c|c|}
\hline $\begin{array}{c}\text { overall correct } \\
\text { probability }\end{array}$ & $\begin{array}{c}\text { without continuous } \\
\text { training }\end{array}$ & $\begin{array}{c}\text { after applying } \\
\text { our algorithm }\end{array}$ \\
\hline Test patient 1 & 0.583 & 0.7844 \\
Test patient 2 & 0.3073 & 0.5601 \\
Test patient 3 & 0.8823 & 0.8921 \\
\hline
\end{tabular}

that the hyperplanes in testing also rotate with respect to the training set position by our algorithm. We compare the correct classification probability with and without continuous training in Table 1. The simulation in the real biomedical data suggests that the decision boundaries shift so that the overall correct classification probabilities increase after applying the Euclidean distance pdf matching algorithm.

\section{CONCLUSION}

This paper proposed a new information theoretic approach for training adaptive system using unlabeled data even after supervised learning is completed. This so called the Euclidean distance pdf matching algorithm utilizes the information of desired signal and unlabeled input signal during the testing phase, which provides a straightforward method to adjust system weights for a better performance. The method requires constant a prior probability for each class during both training and testing, which may be unrealistic in many applications. The simulations on an artificial data set and on real biomedical data suggest that the Euclidean distance pdf matching algorithm can improve classification performance when the a priori hypothesis is met.

\section{ACKNOWLEDGMENT}

This work was supported in part by the National Science Foundation under grant ECS-0300340. We thank Dr. Michael Okun for letting us use the data set collected in the University of Florida Movement Clinic.

\section{REFERENCES}

[1] S. Haykin, Neural Networks: A Comprehensive Foundation, 2nd edition, New Jersey, Prentice Hall, 1999.

[2] B. Widrow,S.D.Stearns, Adaptive Signal Processing, New Jersey, Prentice Hall, 1985.

[3] A.Gammerman, V. Vapnik and V. Vowk, "Learning by Transduction," Proceedings of the Conference on Uncertainty in Artificial Intelligence, pp. 148-155, 1998.

[4] C. Saunders, A.Gammerman and V. Vowk, "Transduction with Confidence and Credibility," Proceedings of the Conference on Uncertainty in Artificial Intelligence, pp. 722-726, 1999.

[5] B. Novak, "Use of Unlabeled Data in Supervised Machine Learning," Proceedings of the Conference on Data Mining and Warehourses, pp. 834 846, Sept./Oct. 2004

[6] A. Blum and T. Mitchell, "Combining Labeled and Unlabeled Data with Co-Training," Proceedings of the Conference on Compuational Learning Theory, pp. 92-100, 1998.

[7] K. Nigram, A. McCallum, S. Thrum and T. Mitchell, "Text Classification from Labeled and Unlabeled Documents using EM," Machine Learning Theory,vol. 39, no. 2,pp. 103-134, 2000.

[8] M. Belkin, P. Niyogi and V. Sindhwani, "Manifold Regularization: A Geometric Framework for Learning from Examples," Technical Report TR-2004-06,Department of Computer Science, University of Chicago, Chicago, Illinois.

[9] D. Erdogmus, Y. Rao and J. C. Principe "Supervised Training of Adaptive Systems with Partially Labeled Data," Proceedings of the International Conference on Accoustic, Speech and Signal Processing,Philadelphia, Pennsylvania, 2005.

[10] J. C. Principe, J. W. Fisher and D.Xu "Information Theoretic Learning," in Unsupervised Adaptive Filtering, S. Haykin Editor, New York, Wiley,pp. 265-319, 2000.

[11] N. Cristianini and J. Shawe-Taylor An Introduction to Support Vector Machines, Cambridge University Press, 2000.

[12] E. Parzen "On Estimation of a Probability Density Function and Mode," in Time Series Analysis Papers, San Diego, Holden-Day, 1967.

[13] D. Erdogmus, J. C. Principe and K. E. Hild, "On-Line Entropy Manipulation: Stochastic Information Gradient," Signal Processing Letters, vol. 10 , no. 8, pp. 242-245, Aug. 2003.

[14] J. Sanchez, J. Pukala, J. C. Principe, F. Bova and M. Okun, "Linear Predictive Analysis for Targeting the Basal Ganglia in Deep Brain Stimulation Surgeries," Proceedings of the Conference on 2nd Int IEEE Workshop on Neural Engineering, Washington 2005. 